\title{
Development of a 3D Hair Simulator to Support a Hairdresser's Haircut
}

\author{
Shota SATO*, Hiroshi YokoYAmA**, Yoichi KageYAMA*, Chikako ISHIZAWA*, \\ Makoto NISHIDA*** and Kazuharu KONISHI ${ }^{\star \star * *}$ \\ * Department of Mathematical Science and Electrical-Electronic-Computer Engineering, Graduate School of Engineering \\ Science, Akita University, 1-1, Tegata Gakuen-machi, Akita-shi, Akita 010-8502, Japan \\ ${ }^{\star *}$ Center for Information Technology and Management, Akita University, \\ 1-1, Tegata Gakuen-machi, Akita-shi, Akita 010-8502, Japan \\ *** The Open University of Japan, 1-1, Tegata Gakuen-machi, Akita-shi, Akita 010-8502, Japan

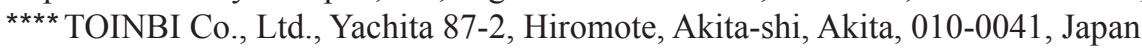 \\ E-mail:kageyama@ie.akita-u.ac.jp
}

\begin{abstract}
While getting a haircut at a hairdresser's, a customer may be unable to effectively communicate his or her hair-related needs. Additionally, the hairdresser often does not share an accurate final image of the customer's hairstyle even with the use of a hair catalog. Head shapes differ for each individual, which implies that the customer's head shape would be different from that of a catalog model. Often, the customer's expectations of the desired hairstyle and the practical results are different. If the final image of the customer's haircut is presented using a 3D hair simulator beforehand, it would facilitate the sharing of hair-related needs between the customer and the hairdresser, thereby improving customer satisfaction. In our previous study, we proposed a basic simulation method that took into consideration the head model generation method and the hairdresser's cutting technique. However, the study did not adequately consider the operability, such as the adjustment of hair in the simulator. Therefore, in this study, we developed a 3D hair simulator to provide support for hairdressers. In particular, we improved the interface for adjusting hair length and evaluated the operability improvement.
\end{abstract}

Keywords : 3D hair simulator, Hairdressers, Hairdresser's cutting technique

\section{INTRODUCTION}

Figure 1 shows an example of the communication while performing a haircut at a hairdresser's. A customer may be unable to effectively communicate his or her hair-related needs to a hairdresser. In addition, the hairdresser does not share an accurate final image of the customer's hairstyle even with the aid of a hair catalog. Head shapes differ for each individual, which implies that the customer's head shape would be different from that of a catalog model. Often, the customer's expectations of the desired hairstyle and the results are different. If the final image of the customer's haircut is presented using a 3D hair simulator beforehand, it would be easy for the customer to share his or her hair-related needs with the hairdresser, thereby improving customer satisfaction.

Conventional studies on hair simulators aimed at reproducing the cut feeling [1], and hair styling for each stratified area obtained by dividing the head horizontally [2]. In addition, studies have been conducted to develop a 3D model of the human face [3] [4]. However, these studies had limitations, namely, inadequate consideration of the inability to reflect the cutting technique of a hairdresser. Therefore, in order to perform a more practical hair simulation, it is necessary to simulate a hairdresser's cutting technique.

In this study, attention was paid to the interface of the hair simulator; its operability and appearance were examined.

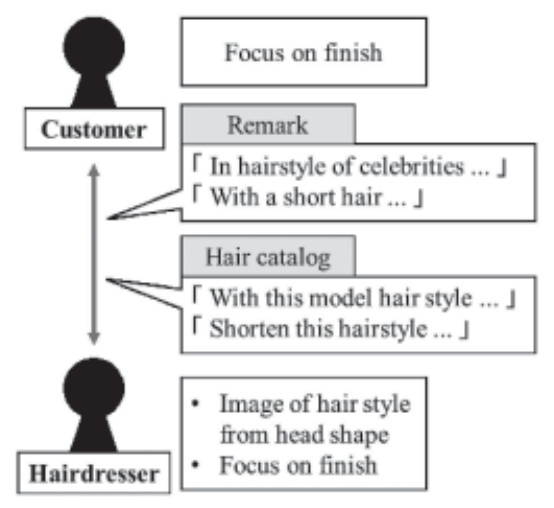

Figure 1 Communication while performing a haircut at a hairdresser's

Specifically, operability was improved by improving the interface related to: the selection of the hair length, the investigation into the type and the length of the bangs, and generating the bangs that match the client's image of the hairstyle.

\section{SPECIFICATIONS OF 3D HAIR SIMULATOR}

\subsection{Actual haircut}

Figure 2 shows the flowchart of cutting hair at a hairdresser's. Figure 3 shows an example of the head area. Figure 4 shows an example of the extent of hair that is easy to cut. First, the 


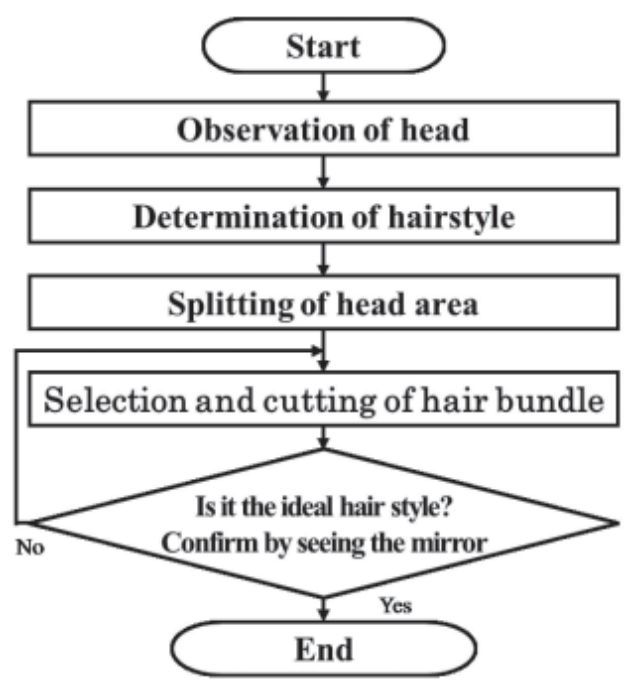

Figure 2 Flowchart of cutting hair at a hairdresser's

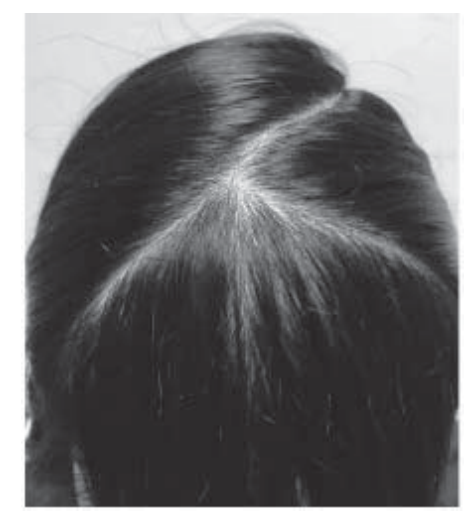

Figure 3 Example of the head area

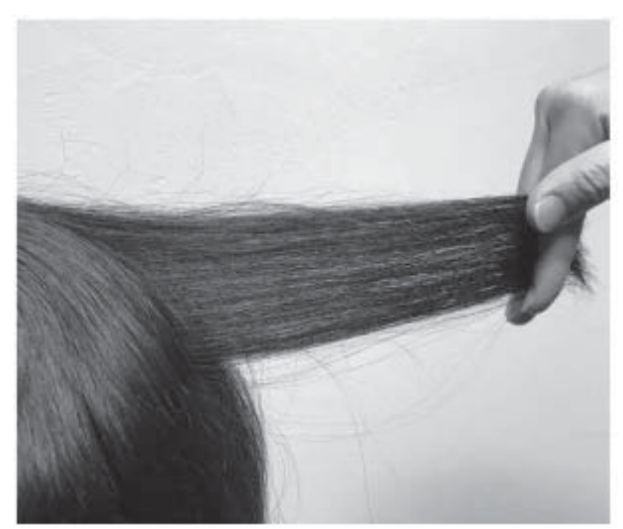

Figure 4 Example of the extent of hair that is easy to cut

hairdresser observes the characteristics of the head of the customer and determines the hairstyle desired by the customer by using a hair catalog or using words to describe the hairstyle. Next, as shown in Figure 3, the head area is divided based on the characteristics and roles of the hair (bangs: face impression changes, back hair: weight and depth). As shown in Figure 4, the extent of hair to be cut is manually picked up and cut. Finally, the mirror is used to check whether the hairstyle is as desired by the customer. By following

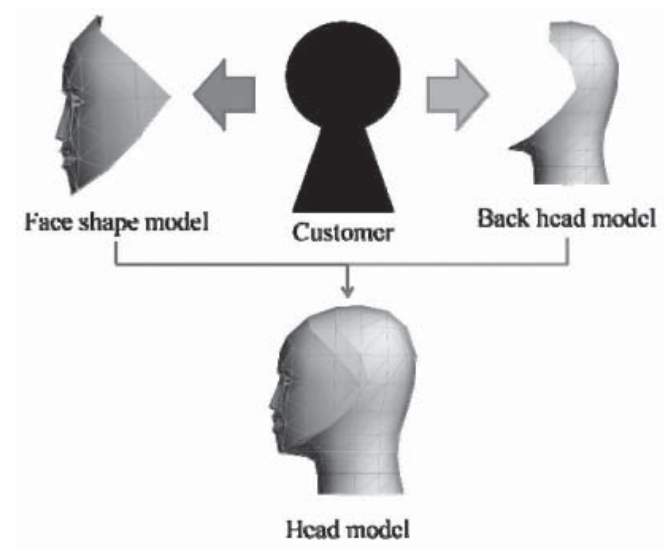

Figure 5 Flow of the head model generation

the steps above, a haircut is performed.

\subsection{System overview}

The specifications of the 3D hair simulator in our previous study [5] include the following processes.

2.2.1 Generation of a head model: Figure 5 shows the flow of the head model generation. First, a picture of the facial region is captured using Kinect [6], and the feature points in the region are extracted. Next, by focusing on the extracted feature points, a model of the face shape is generated. At the same time, a 3D model is generated using the coordinates acquired by the infrared camera. Subsequently, we combine the generated face shape model with the fixed back head model prepared beforehand to generate a complete head model. However, given that the shape of the occipital region differs for each individual, the occipital region of the head model is divided and shaped using a slider.

2.2.2 Hair adjustment: Hair is applied to the head model, and the head area is divided into 18 parts based on the different sections of hair. For each section of hair, the length and cutting method can be adjusted using a slider.

2.2.3 Simulation flow: First, we construct the hair strips and simulate them by using physical calculations. Next, after the simulation, a hair generation process is performed for the space between the hair. Finally, a display polygon is generated, and a texture is applied to the polygon. The hairstyle obtained is presented to the customer.

\subsection{System issues}

The 3D hair simulator in our previous study [5] had the following issues.

(1) Due to the fact that the hair length is set to the maximum value in the initial state, it is necessary to adjust the hairstyle as desired by the customer.

(2) Given that the length of the hair is not displayed numerically, it is difficult to capture the exact length.

\section{PROPOSED METHOD}

\subsection{Setting the Max value of the hair}

Figure 6 shows the screen for setting the Max value of hair. By entering a numerical value in the text box, a user can set the maximum value of bangs and other hair. In addition, it is possible to select the maximum value with three buttons for "long", "middle", and "short" presets. Furthermore, given that it is possible 


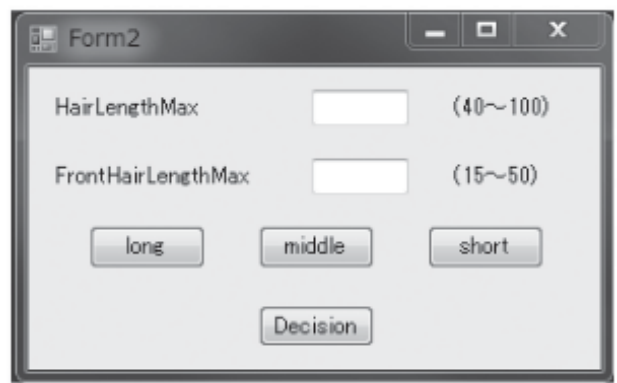

Figure 6 Flowchart of cutting hair in the hairdressing room

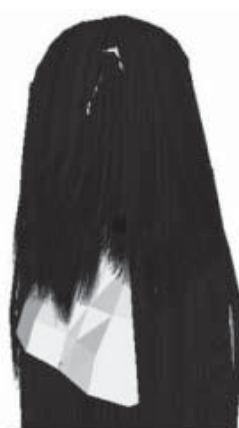

(a) Before

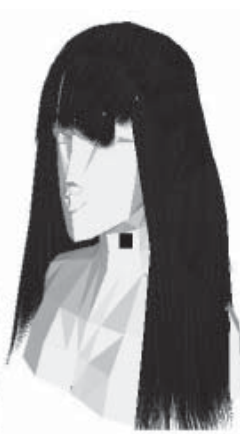

(b) Long hair

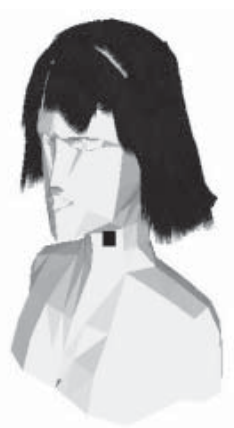

(c) Short hair to determine the approximate length of the hair based on the current hairstyle of the customer who came into the hairdressing room, the max value of the hair can be changed. In addition, we considered the opinions of experts and selected the maximum value. Since the length of the hair can be roughly grasped when customers come to the hairdresser, the maximum value can be set by using the box or the preset button, and the simulation can be performed only by adjusting the hair style.

\subsection{Initial setting of hairstyle}

Figure 7 shows the outline of the head area, and Table 1 lists the initial values of hair length in each section. Figure 8 shows an example of a hairstyle before and after the initial value change. As shown in Figure 7, the head area is divided based on the characteristics of the hair, and an initial value is set for 3D hair models as listed in Table 1. By considering three different lengths "long hair," "medium hair," and "short hair," the corresponding

Table 1 Initial value of hair length in each section

\begin{tabular}{|r|r|r|r|r|}
\hline \multirow{2}{*}{ Head region } & \multicolumn{4}{|c|}{ Hair length (cm) } \\
\cline { 2 - 5 } & $\begin{array}{c}\text { Old initial } \\
\text { value }\end{array}$ & short hair & $\begin{array}{c}\text { medium } \\
\text { hair }\end{array}$ & long hair \\
\hline I1 & 30 & 7 & 8 & 9 \\
\hline I2 & 30 & 10 & 12 & 12 \\
\hline I3 & 50 & 10 & 14 & 20 \\
\hline I4 & 100 & 15 & 27 & 40 \\
\hline I5 & 100 & 15 & 27 & 40 \\
\hline E1 & 30 & 5 & 8 & 9 \\
\hline E2 & 50 & 8 & 14 & 20 \\
\hline E3 & 50 & 8 & 14 & 20 \\
\hline E4 & 100 & 15 & 27 & 40 \\
\hline
\end{tabular}

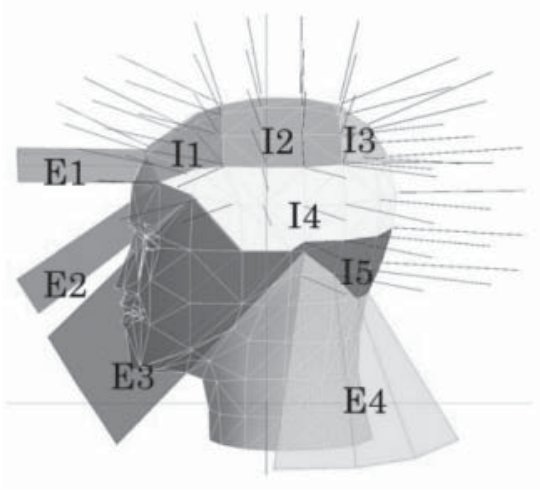

Figure 7 Outline of head area
Figure 8 Example of a hairstyle before and after the initial value change

length of hair of 3D hair models is set by selecting a hairstyle from the selection screen. By selecting the hairstyle most similar to that of the customer, it is possible to simulate the hairstyle that the customer expects. Also, it is expected to reduce the number of adjustment procedures by choosing a preset hair style that is close to the current hairstyle of the customer.

\subsection{Changing the appearance of the head model}

Figure 9 shows the head model of the proposed method and the head model of the conventional method. In the head model of the conventional method, irregularities were conspicuous. Therefore, we modified the texture of the head model and used multiple lighting in OpenGL [7]. By applying these effects, it is possible to make the shadow inconspicuous and make irregularities of the face area inconspicuous.

\subsection{Hair adjustment using boxes and sliders}

Figure 10 shows the hair adjustment slider. It was difficult to know the hair length of each hair section unless skilled hairdresser. There, by displaying the numerical value adjusted by the slider, it is easy to capture the exact length. Additionally, by synchronizing the box and the slider, it is possible to adjust the length of the hair by entering a numerical value directly in the box.

\subsection{Study on the type and shape of bangs}

Figure 11 shows the bangs setting screen. Since bangs affect the person's impression formation, bangs require careful image sharing. In this study, six kinds of "right-side parting", "left-side parting", "center parting", "hair upward", "straight-cut bangs", and "M-bangs" were set as the type of bangs. The bangs were selected after considering the opinions of experts.

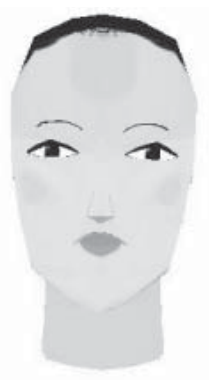

(a) The proposed method

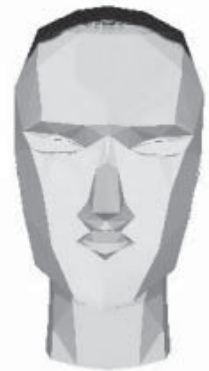

(b) The conventional method
Figure 9 Example of the head area 


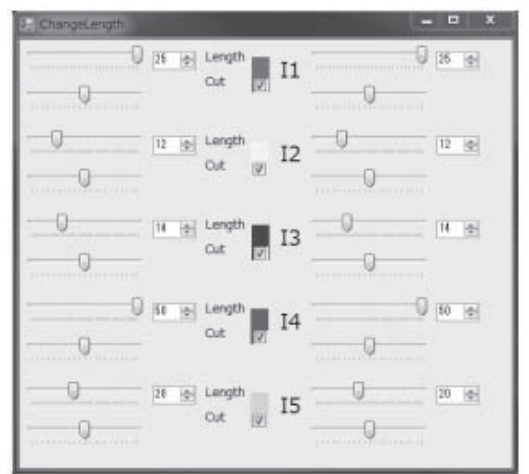

Figure 10 Hair adjustment slider

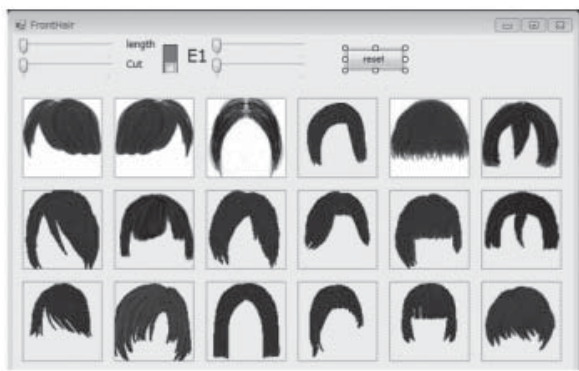

Figure 11 Bangs setting screen

By selecting from the bangs of a total of 18 types (three types of six types of forelocks) shown in the selection screen of Figure 11 for the output model, data corresponding to the forelock is read and reflected on the hair of $3 \mathrm{D}$ model. As a result, it becomes possible to perform a simulation with the customer's favorite forelock.

\section{EXPERIMENT TO EVALUATE USEFULNESS}

Figure 12 displays the experiment environment. In order to determine the usefulness of the proposed interface, an evaluation experiment was conducted. The subjects were the students (seven men and nine females in their twenties) and the hairdresser (one male in their forties). In addition, the students participated in the experiment as customers. The experiment procedure is as follows:

(1) Explain the operation of the hair simulator in the conventional method [5] to a subject.

(2) The subject operates the conventional hair simulator.

(3) Explain the operation of the hair simulator in the proposed method (see section 3 ) to a subject.

(4) The subject operates the proposed hair simulator.

(5) The subject fills in the evaluation questionnaire.

Table 2 lists the items of the questionnaire and Table 3 lists the response options. For the evaluation, question is done in 5-point

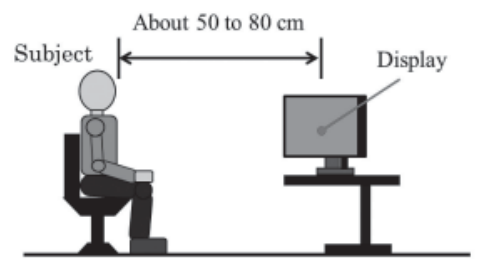

Figure 12 Experiment environment
Table 2 Items of the questionnaire

\begin{tabular}{|c|c|}
\hline $\begin{array}{c}\text { Question } \\
\text { No. }\end{array}$ & Item Description \\
\hline $\mathbf{n}$ On the hair simulator of the proposed method \\
\hline 1 & Is the appearance of the front bangs good? \\
\hline 2 & Is it easy to operate the bangs selection screen? \\
\hline 3 & Are the types of bangs adequate? \\
\hline 4 & Does the model look good? \\
\hline 5 & Does the resulting model make it easier to visualize the hair style? \\
\hline 6 & Is the maximum setting screen easy to operate? \\
\hline 7 & Is the of hair styles adequate? \\
\hline 8 & Is it easy to adjust the hair length by setting the maximum value? \\
\hline 9 & Is it easy to adjust the hair length by selecting the initial value? \\
\hline 10 & Do you really want to use it in at a hairdresser's? \\
\hline $\mathbf{R e g a r d i n g}$ the hair simulator of the conventional method \\
\hline 11 & Is the appearance of the front bangs good? \\
\hline 12 & Are the types of bangs adequate? \\
\hline 13 & Does the model look good? \\
\hline 14 & As it easy to adjust the hair from the initial value to the \\
\hline 15 & Does the resulting model make it easier to visualize the hair style? \\
\hline 16 & Are types of hair styles adequate? \\
\hline 17 & Is it easy to adjust the hair whose maximum value is fixed? \\
\hline 18 & Please freely describe what you felt when looking at the system \\
\hline 19 & \\
\hline
\end{tabular}

Table 3 Response options for the questionnaire

\begin{tabular}{|l|l|l|l|l|}
\hline 5. Excellent & 4. Good & 3. Average & 2. Below average & 1. Poor \\
\hline
\end{tabular}

scale of "Excellent", "Good", “Average”, "Below average", "Poor". Incidentally, the data used in the study was acquired in accordance with the ethics rules regarding human subjects at Akita University, Japan.

\section{RESULTS OF EXPERIMENT}

Table 4 lists the results of the evaluation and the average scores of each question. The Question No. in Table 2 and Table 4 correspond to each other. Also, it is assumed that it is higher than 4.0 and lower than 2.5. Compared questions 1 and 2 with questions 11 and 12 , the proposed method gained a higher evaluation than the conventional method. In addition, while the evaluation of question 13 was as low as 1.5 , the evaluation of question 3 obtain a high value of 4.3 among all students and 4.7 for the female average. It became clear that the desired hairstyle can be displayed by the proposed method, indicating that the selection was also sufficient. In fact, the evaluation revealed than an image of the customer was easy to obtain. Although the evaluation of question 14 was 2.5 , the results of question 4 revealed a judgement that the appearance of the model has improved, given that the overall average feedback of the students and the hairdresser was 4.0. While the evaluation of question 18 was as low as 2.1, the average of questions 7,8 and 9 and the evaluation of the hairdresser revealed a high value of 4.0 or more.

That is, the conventional method had a problem that the initial value was the maximum value when adjusting the hair. Conversely, with the proposed method, it is possible to set the maximum value from the setting screen of the max value of the hair and select the initial value of the hair. Therefore, it is possible to grasp the 
Table 4 Results of the evaluation and average scores of each question

\begin{tabular}{|c|c|c|c|c|c|}
\hline \multirow{2}{*}{$\begin{array}{l}\text { Ques- } \\
\text { tion No. }\end{array}$} & \multirow[b]{2}{*}{ Item Description } & \multicolumn{4}{|c|}{ score } \\
\hline & & \begin{tabular}{c|}
$\begin{array}{c}\text { male } \\
\text { students }\end{array}$ \\
\end{tabular} & \begin{tabular}{|c|} 
female \\
students
\end{tabular} & \begin{tabular}{|c|c}
$\begin{array}{c}\text { all } \\
\text { students }\end{array}$ & \\
\end{tabular} & \begin{tabular}{c|} 
hair- \\
dresser
\end{tabular} \\
\hline \multicolumn{6}{|c|}{ On the hair simulator of the proposed method } \\
\hline 1 & $\begin{array}{l}\text { Is the appearance of the front bangs } \\
\text { good? }\end{array}$ & 3.7 & 4.0 & 3.8 & 3. \\
\hline 2 & $\begin{array}{l}\text { Is it easy to operate the bangs } \\
\text { selection screen? }\end{array}$ & 3.7 & 4.9 & 3.8 & 2.0 \\
\hline 3 & Are the types of bangs adequate? & 3.9 & 4.7 & 4.3 & 2.0 \\
\hline 4 & Does the model look good? & 4.3 & 3.8 & 4.0 & 4.0 \\
\hline 5 & $\begin{array}{l}\text { Does the resulting model make it } \\
\text { easier to visualize the hair style? }\end{array}$ & 4.6 & 4.0 & 4.3 & 2.0 \\
\hline 6 & Are the types of hair styles adequate? & 3.7 & 3.8 & 3.8 & 2.0 \\
\hline 7 & $\begin{array}{l}\text { Is the maximum setting screen easy to } \\
\text { operate? }\end{array}$ & 4.6 & 4.7 & 4.6 & 4.0 \\
\hline 8 & $\begin{array}{l}\text { Is it easy to adjust the length of hair } \\
\text { by setting the maximum value? }\end{array}$ & 4.1 & 4.6 & 4.4 & 4.9 \\
\hline 9 & $\begin{array}{l}\text { Is it easy to adjust the length of hair } \\
\text { by selecting the initial value? }\end{array}$ & 4.9 & 4.6 & 4.7 & 4.0 \\
\hline 10 & $\begin{array}{l}\text { Do you really want to use it in at a } \\
\text { hairdresser's? }\end{array}$ & 3.6 & 3.9 & 3.8 & 2.0 \\
\hline \multicolumn{6}{|c|}{ Regarding the hair simulator of the conventional method } \\
\hline 11 & $\begin{array}{l}\text { Is the appearance of the front bangs } \\
\text { good? }\end{array}$ & 3.4 & 3.0 & 3.2 & 1.0 \\
\hline 12 & $\begin{array}{l}\text { Is it easy to operate the bangs } \\
\text { selection screen? }\end{array}$ & 2.4 & 2.8 & 2.6 & 3.0 \\
\hline 13 & Are the types of bangs adequate? & 1.4 & 1.6 & 1.5 & 1.0 \\
\hline 14 & Does the model look good? & 2.1 & 2.8 & 2.5 & 1.0 \\
\hline 15 & $\begin{array}{l}\text { Does the resulting model make it } \\
\text { easier to visualize the hair style? }\end{array}$ & 3.3 & 2.8 & 3.0 & 1.0 \\
\hline 16 & Are the types of hair styles adequate? & 2.0 & 2.4 & 2.3 & 1.0 \\
\hline 17 & $\begin{array}{l}\text { Is it easy to adjust the hair whose } \\
\text { maximum value is fixed? }\end{array}$ & 2.6 & 2.3 & 2.4 & 2.0 \\
\hline 18 & $\begin{array}{l}\text { Is it easy to adjust the hair from the initial } \\
\text { value to the maximum value? }\end{array}$ & 2.3 & 1.9 & 2.1 & 2.0 \\
\hline 19 & $\begin{array}{l}\text { Do you really want to use it at a } \\
\text { hairdresser's? }\end{array}$ & 2.4 & 2.6 & 2.5 & 1.0 \\
\hline
\end{tabular}

approximate maximum value based on the hair length of the customer and better match the customer's needs by being able to more easily adjust the hair and select a hair style. These are the main reasons why we believe that the proposed method obtained higher evaluations.

The results above suggest that the operability and appearance of the 3D hair simulator proposed in this study have been improved when compared to the conventional method.

\section{CONCLUSION}

In order to improve operability, this paper examined the operability and appearance of the simulator with the aim of generating bangs that match the images of hairstyles of customers and hairdressers. Specifically, we proposed a bangs adjustment method by setting the initial value of the length of the hair using a slider hair adjustment method, box operation, and option to select the type of bangs. The results obtained are summarized below:

1. By displaying the hair settings numerically, it was clarified that a detailed length can be grasped when adjusting hair.

2. While adjusting the bangs, it was clear that it is possible to select various types of bangs.

3. Compared with the conventional method, the hair simulator of the proposed method clarified that hair adjustment is easy by setting the maximum value of the hair length and the initial value of the hair length.

4. By improving the appearance of the head model, it became clear that the image of the hair style becomes easier to visualize based on the head model.

\section{Acknowledgements}

The authors thank TOINBI Co., Ltd. for their help in conducting the experiments.

\section{References}

[1] Motoki KONDO.; Jun MITANI.; Yukio FUKUI.; Yoshihiro KANAMORI., "A method for generating an illustration for haircut instruction combined with physician simulation" The Proceedings of the 73rd National Convention of IPSJ. 6Z-6, 121-122 (2011).

[2] Lieu-Hen CHEN.; Santi SAEYOR.; Hiroshi DOHI.; Mitsuru ISHIZUKA., "A system of 3D hair style synthesis based on the wisp model" VISUAL COMPUTER 15, 4, 159-170 (1999).

[3] Katsuhiro KITAJIMA.; Yasuhiro AKAGI.; Akira YAMAUCHI.; Naoki OKAZAWA.; Yasukazu HIGUCHI., "A study on facial modeling based on the GFFD method" Journal of The Japan Society for Precision Engineering. 74, 8, 883-890 (2008).

[4] Davide MINNAI.; Taisei ITO.; Ryo OHKAWA.; Takahumi KOTSUKA.; Hitoshi TAMURA., "Creation of a $3 \mathrm{~d}$ face model using a RGB-Depth camera device" The Proceedings of the 74rd National Convention of IPSJ. 2R-3, 245-246 (2012).

[5] Keitaro TANAMURA.; Hiroshi YOKOYAMA.; Makoto NISHIDA.; Yoichi KAGEYAMA., "Development of Hair Simulator using a Depth Camera" Heisei 26th First Information Processing Society Tohoku Branch Study Group. Session 2, 8p (2014).

[6] Kinect for Windows Web Site., http://www.microsoft.com/en-us/kinectforwindows/ (accessed 2018.01.01)

[7] OpenGL formulation committee., "OpenGL Programming Guide Original edition 5th edition" Pearson Japan K.K. (1993) 